\title{
Powerful Learning with Computational Thinking: Our Why, What, and How of Computational Thinking
}

\section{Digital Promise}

\section{March 2021}
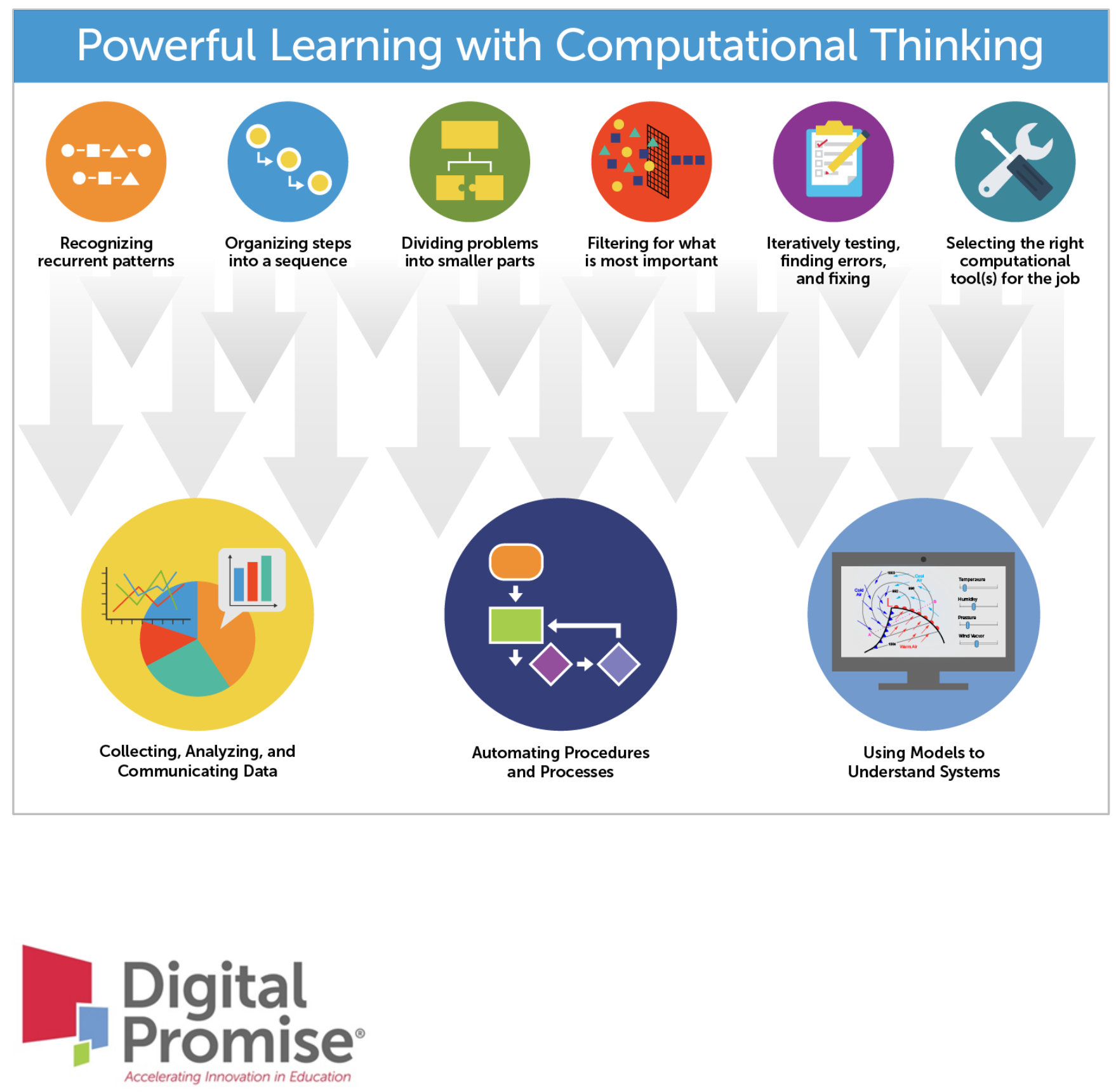


\section{Suggested Citation}

Digital Promise. (2021, March). Powerful learning with computational thinking: Our why, what, and how of computational thinking. Digital Promise. https://doi.org/10.51388/20.500.12265/115

\section{Acknowledgments}

This report was developed under the guidance of Kelly Mills, Quinn Burke, Merijke Coenraad, Ximena Dominguez, Emi Iwatani, Danae Kamdar, Tiffany Leones, Kevin McElhaney, Jeremy Roschelle, Pati Ruiz, and Josh Weisgrau of Digital Promise. Carly Chillmon supported the team with writing, editing, and advice.

\section{Contact Information}

Digital Promise:

Washington, D.C.:

1001 Connecticut Avenue NW, Suite 935

Washington, DC 20036

San Mateo, CA:

2955 Campus Dr. Suite 110

San Mateo, CA 94403

https://digitalpromise.org/

(c)

This work is licensed under a Creative Commons Attribution NonCommercial 4.0 International License. 


\section{Why We Focus on Computational Thinking}

Educators aim to prepare students to pursue their own best possible futures. Students must be prepared to be problem solvers, team leaders, collaborators, communicators, creative thinkers, and more. Toward this end, we support them by weaving dynamic opportunities to learn and apply these skills into the teaching of core academic subject matter like English language arts (ELA), mathematics, science, and social studies. For example, integrating teamwork, engaging in project-based learning, or applying a design process is now commonplace across academic subject areas.

As students learn the skills of problem-solving, leading teams, communicating, and creating innovations, they will need to leverage the unique affordances of computers to successfully engage them. In particular, their use of computers will move beyond consuming information or doing routine tasks like reading, writing, or presenting. For example:

- In solving a social studies problem about water rights in different states, students may need to create a data visualization to communicate essential information to key stakeholders.

- In leading a scientific team to solve a problem about plant growth, students may need to create or modify a computer simulation of plant growth.

- To communicate effectively in a mathematics classroom, students may use a sequence or flowchart to express their strategy for tackling a mathematical challenge.

- To analyze how an author uses specific words, students could create a computer algorithm that identifies different ways a certain word is used in the text.

To prepare students for our increasingly computational world, they will need opportunities to go beyond using a computer to gather information, organize, write, or present. Students will need learning opportunities to create, modify, customize, adapt, or otherwise manipulate a computer in support of their goals (NASEM, 2021). These opportunities to learn will be important to all students-not only the ones who will eventually study computer science or enter the information technology industry. To prepare students for the ways computing is becoming integrated into seemingly every aspect of society, $\mathrm{K}-12$ students need opportunities to apply computing skills across ELA, math, science, social studies, and more.

Computational thinking (CT) has been used to describe this kind of learning. Yet, in our experience, many educators find this term mystifying. How can we concisely talk about opportunities to learn how to use computers constructively as part of our learning about problem-solving, collaborating, communicating, and creating? How can we specify opportunities to learn these skills that integrate with core academic subject matter? How can we clarify what it looks like to provide these learning opportunities inclusively to all students, not just those who decide to pursue coding, careers in IT, or further study of computer science? 
In this paper, we explain how our team at Digital Promise, working closely with innovative districts and teachers, has examined the complex jargon of "computational thinking," "computer science," and "coding" to make the ideas more concrete to practitioners for teaching, design, and assessment. We describe three powerful ways of using computers that integrate well with academic subject matter and align to our goals for students: (1) collecting, analyzing, and communicating data; (2) automating procedures and processes; and (3) using models to understand systems. We describe a set of underlying skills that are the building blocks for all three powerful uses.

We also articulate our four-part strategy for working with teachers, schools, and districts to do this work. Teachers, schools, and districts are already integrating problem-solving, teamwork, and design across the curriculum. We describe how practitioners can take CT to the next level by:

- Focusing on PreK-8 integration of computing and engaging teachers with professional learning opportunities to promote this integration

- Committing at the district level to support learning opportunities that are cumulative, consistent, and competency-based

- Designing for inclusive participation of students historically marginalized from computing

- Engaging teachers, families, and designers in ongoing participatory and iterative design to improve learning opportunities

Brief examples of our work in practice illustrate how four districts have engaged with us to move forward.

\section{What We Mean by Computational Thinking}

We support teachers in identifying ways to leverage computational thinking to enhance their students' learning. Drawing from both learning sciences research and feedback from educators and district leaders, we define CT to be a specific set of practices and skills. Figure 1 represents the relationships among these aspects of $C T$, enabling teachers in any subject to find productive intersections among these practices and skills and to support students in what they are expected to know and know how to do. 
Figure 1

Powerful Learning with Computational Thinking

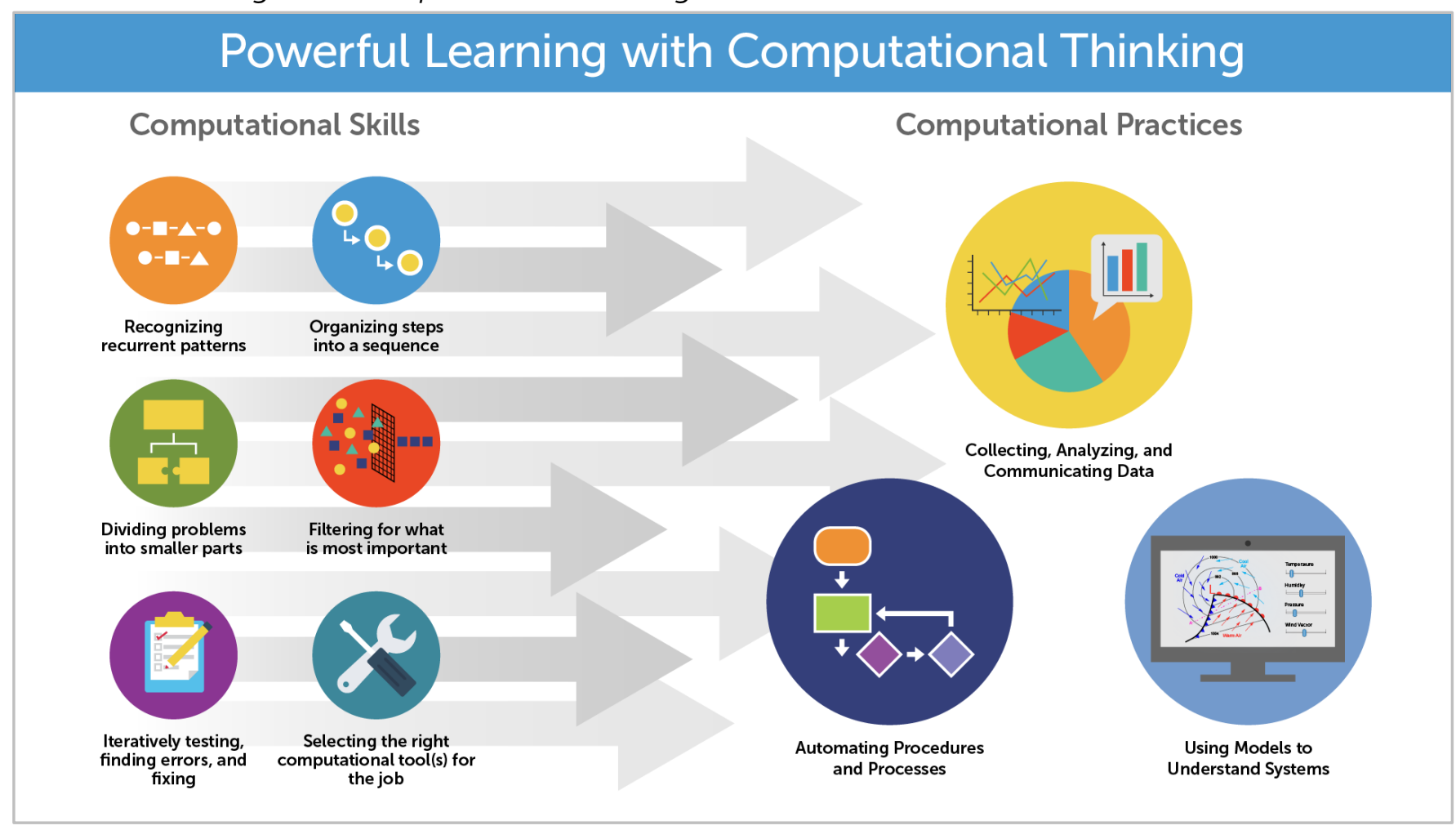

Computational skills are the cognitive processes necessary to engage with computational tools to solve problems. Computational practices combine many computational skills to solve an applied problem.

The language in the figure intentionally excludes technical terminology that can act as a barrier for practitioners that do not have a background in computer science. Instead, we operationalize concepts for educators through everyday language. For example, computational skills include

- dividing problems into smaller parts, or problem decomposition

- filtering aspects of a problem for what is most important, otherwise known as abstraction

- iteratively testing, finding errors and fixing them, more commonly known as debugging

- organizing steps in a sequence, or algorithmic thinking

- recognizing recurrent patterns

- selecting the right computational tool(s) for the job

Computational practices are the application of these foundational skills, which often result in a computer program, a data visualization, or computational model that could be used to solve related problems in the future. For example, when considering the amount of food waste within their lunch at school, students might collect, analyze, and communicate data. 
Specifically, they could collect data about how much food is thrown away, what is part of the food waste, and the reasons students give for throwing away food. Then, they may filter for what is most important and divide problems into smaller parts to define categories of food that is thrown away. Finally, students can recognize recurring patterns to make conclusions about the food waste in their school and share those conclusions through a data visualization. Depending on the amount of data they collect, students might need to select the right computational tool to help them filter, analyze, or present their data. Later, they might be able to use this tool to analyze food waste in a slightly different situation, for example, at their school's sporting or performance events.

For each practice, we have developed "look fors," which are professional learning tools that prompt teachers to identify students' productive use of CT practices as part of their learning activities. The "look fors" operationalize the CT practices and underlying skills to help teachers highlight where CT is or could be happening within their classrooms. Here we present common "look fors" to help illustrate and define CT practices and skills.

\section{Look Fors}

Teachers will know students are engaging in computational thinking practices because they may observe the following student actions:

\section{Collecting, Analyzing, and Communicating Data}

- Designing an experiment utilizing computational tools to collect data

- Collecting data that can be quantified

- Manipulating data with data moves

- Describing relationships between variables

- Using data to make predictions

- Identifying bias in data collection and reporting

- Considering if/how data sources are comparable

- Designing a visual representation of data

- Selecting design features to communicate to a particular audience

\section{Automating Procedures and Processes}

- Decomposing problems or tasks

- Identifying essential steps

- Coding

- Identifying and repairing errors

- Considering efficiency

\section{Using Models to Understand Systems}

- Identifying a question to explore using a computational model 
- Setting up multiple and different scenarios to collect data from a computational model

- Making predictions about how the model will behave with different inputs

- Identifying different parts of the system that the model is representing

- Defining relationships between different parts of a system

- Automating relationships between parts of the system with a flowchart or programming/modeling software

- Considering how the model represents the real-world system

- Considering bias in the outputs of a computational model

\section{How We Promote Computational Thinking}

\section{Figure 2}

Digital Promise's Four Main Commitments to Computational Thinking
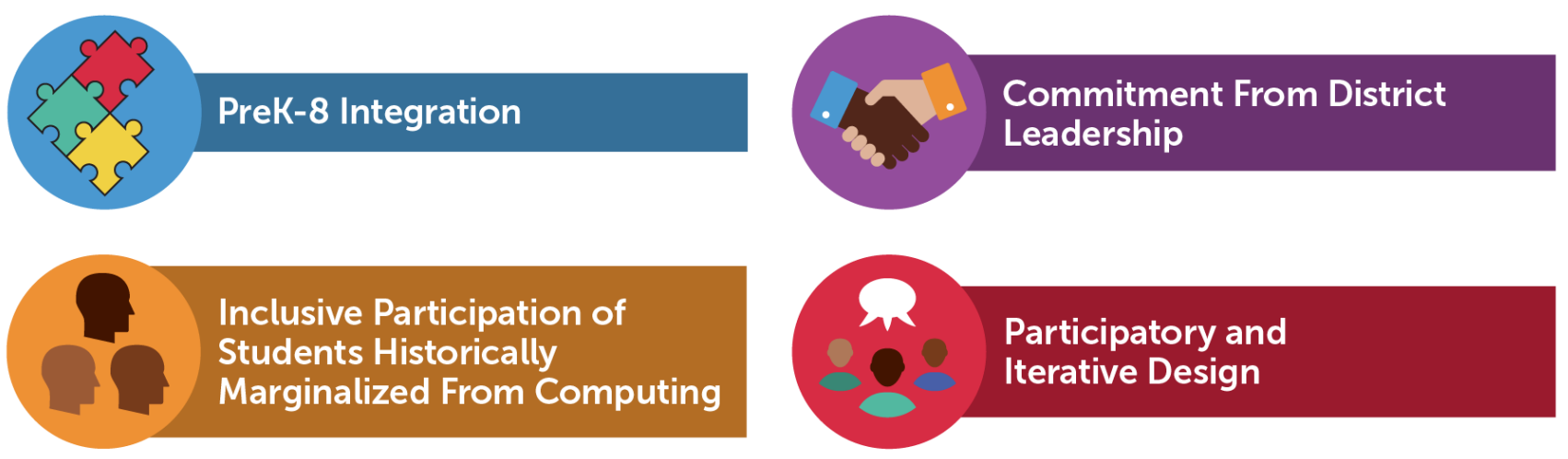

In an internal working group, we reflected on the contributions of seven computational thinking projects our team was concurrently involved with. Through doing so, we recognized that the Digital Promise approach has four main commitments.

\section{Focus on PreK-8 Integration (Rather than Stand-Alone Clubs and Classes)}

Traditionally, computing opportunities have existed only as electives, meant for a select few students. Creating access, particularly for historically minoritized students, often requires integrating $\mathrm{CT}$ into the existing subject matter rather than relying on stand-alone coursework or activities. Infusing CT in ways that are connected to familiar topics enables students to realize their agency with computational problem solving, which may build students' attitudes and confidence toward computing. The interdisciplinary nature of CT has overlapping, synergistic concepts and practices with ELA, social studies, science, math, and more, having 
the potential to enhance learning in each domain (Barr \& Stephenson, 2011; Lee et al., 2020; Nesiba et al., 2015; Settle et al., 2012; Waterman et al., 2020). In this way, integrating CT becomes a value-add, as opposed to an add-on. We have seen the capacity of $\mathrm{K}-8$ teachers to own and translate CT content into their own existing academic disciplines/expertise when provided appropriate support. We have worked with teachers to develop professional learning opportunities and resources that enhance disciplinary learning, increase student agency, and equip students with the skills and habits of mind they need to productively engage in our increasingly computational world.

\section{Commitment from District Leadership (Rather than School-to-School Variability)}

While some of the most dynamic and interesting initiatives in CT often come from grassroots, bottom-up initiatives that start with a single dedicated teacher (or, in some cases, a single adventurous student), for wider adoption across schools and a district, these interventions are rarely scalable or sustainable. Our district partners have played a key role in the design and implementation of computing pathways that align with ongoing initiatives and systems of professional learning to generate buy-in from different stakeholders (e.g., students, parents, teachers, building leaders). It is quite clear: Leadership matters. State-level policies are too far removed from the contexts of school communities and one-off computing interventions (e.g., particular courses, coding events, after-school clubs) do not reach all students and are subject to school-to-school variability. We support districts to develop computing pathways that are deeply integrated into the core curriculum for all K-12 students, providing consistent, cumulative, and context-relevant learning opportunities to every student (Burke et al., 2019; Roschelle et al., 2021). We have worked with districts to identify how CT informs the wider district's mission and their commitment to students and the community and develop sustainable implementation strategies that align with ongoing initiatives.

\section{Design for Inclusive Participation of Students Historically Marginalized from Computing (Rather than Equal Access for All)}

We strive to go beyond access, designing inclusive opportunities for historically marginalized learners. Stereotypes about who should excel in computing lead to inequitable tracking of young women and students of color out of opportunities in computing (Margolis, 2010; Pinkard et al., 2017; Ryoo, 2019). There is a need for educational systems to rethink opportunities available for students to engage in computing to remove systemic barriers. We engage in this work through research to understand conditions that are most engaging to historically marginalized learners and design learning opportunities that make connections to lives, interests, and communities of students furthest from opportunity. We work with districts and teachers that have articulated clear equity challenges in their contexts. With these challenges in mind, we are utilizing noticing activities, empathy interviews (Ruiz et al., 2021), learning sciences research, and analysis of student data to consider the perspectives and experiences of students historically marginalized in computing. Using what teachers learn from their students and existing research, we are collaboratively designing lessons and considering asset-based pathways into computing for all students. 


\section{Engagement in Participatory and Iterative Design (Rather than a Packaged Solution)}

Our CT work is grounded in research-practice partnerships leveraging the expertise and perspectives of multiple stakeholders. Our co-design teams include learning scientists, district leaders, teachers, families, children, curriculum designers, and/or media developers. Through our co-design process, stakeholders have been asked to: (1) share their unique perspectives and insights; (2) brainstorm whether and which CT skills could be consequential for learning in their context; and (3) collaboratively develop (and test) CT integrated learning activities and digital apps (Dominguez et al., 2020). Through this work together, we aimed to identify learning experiences that resonate with families and children. Our goal is to capitalize on children's and families' funds of knowledge (Moll, 2015) and identify activities that could bridge home and school learning. We take an inclusive approach to ensure all stakeholders share their unique perspectives and insights. As with most collaborative processes, we recognized the need to allocate time to promote flexibility and build rapport, cultivating a sense of openness and mutual respect that recognizes everyone's voice and perspective, while also encouraging all stakeholders to feel comfortable and confident in the design process.

\section{Examples of our Work in Practice}

\section{Focus on PreK-8 Integration}

Jessica Bibbs-Fox, a teacher in Compton Unified School District (California), integrated CT data practices into her virtual middle school science class as part of the Computational Thinking Micro-credentials and NGSS project funded by the Carnegie Corporation of New York. Using the COVID-19 pandemic as the anchoring phenomenon, Bibbs-Fox designed a unit in which students examined the accuracy of information related to the virus. She adapted computational thinking resources from Digital Promise to support students with collecting, analyzing, and evaluating data and communicating data.

Bibbs-Fox acknowledged that computational thinking is a skill set that is new to many students. To support her students in

They are starting to see themselves as

scientists and understand the nature of

science as a human endeavor.

- Jessica Bibbs-Fox, teacher,

Compton Unified School District the process, she broke down each step, beginning with having students ask questions based on their curiosities about the pandemic. Bibbs-Fox did not anticipate that many students would struggle to generate questions that could be explored using data. For example, initially, one student posed the question, "Why is the coronavirus so deadly?" which is not readily answered with data. She helped this student to modify the question so that it could be answered using the data available; for example, "Compared to other coronaviruses, is COVID-19 resulting in more deaths?" Next came manipulating the data in spreadsheets, a new and somewhat intimidating skill for many students. However, Bibbs-Fox reported that this activity helped students to resolve a fear of seeing spreadsheets and feel a certain level of pride in working with them. 


\section{Figure 3}

Example of Students Using Data to Answer a Driving Question

\section{Question: What country has the highest number of confirmed cases?}

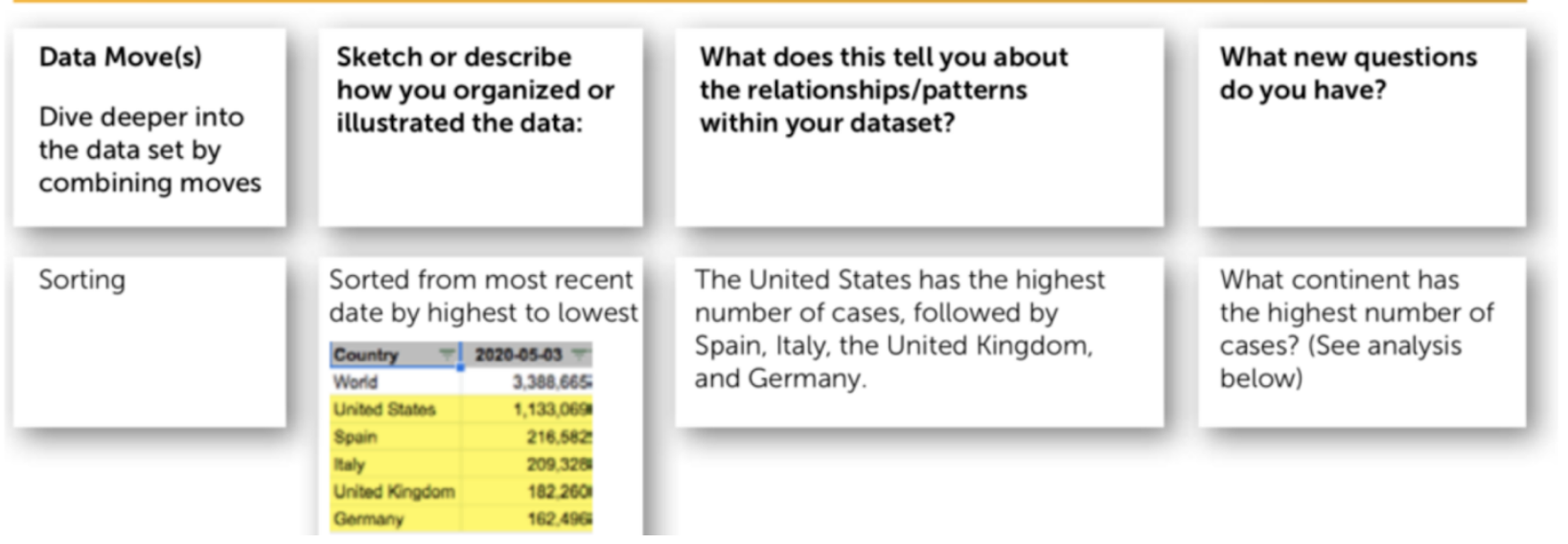

Overall, Bibbs-Fox said this project is the most authentic learning experience she has worked on with her students. She explained, "This project relies on questions that do not have answers readily available to them. Students have to rely on their skills to be successful."

After completing this lesson, Bibbs-Fox earned the Analyzing and Communicating Data micro-credential (pictured below). Digital Promise strives to recognize educators for creating learning experiences where students can build competencies through our micro-credential platform. To earn a micro-credential, teachers submit evidence of student work from classroom activities where they have applied one of the CT practices as documentation of lesson planning and reflection.

\section{Figure 4}

Digital Promise's Computational Thinking Micro-credentials

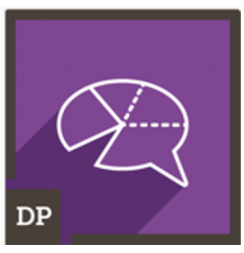

Analyzing \&

Communicating Data

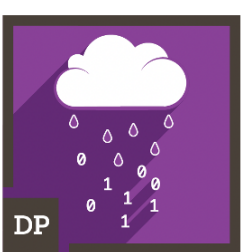

Collecting \& Structuring Data

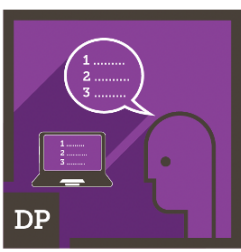

Creating Algorithms

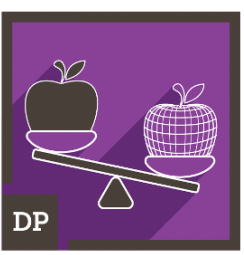

Creating Computational Models

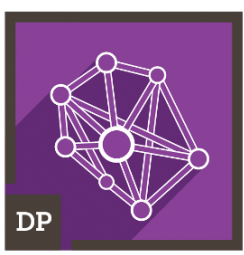

Understanding Systems

- Highlighted resources:

- Computational Thinking for Next Generation Science Toolkit

- Computational Thinking Micro-credentials 


\section{Commitment from District Leadership}

Talladega County Schools is a rural district in central Alabama. Over the past three years, they have participated in the National Science Foundation-funded project, Developing Inclusive K-12 Computing Pathways for the League of Innovative Schools, working in a researchpractice partnership to identify opportunities and develop the capacity to integrate $\mathrm{CT}$ in $\mathrm{K}$ 12 disciplinary learning. Dr. Brooke Morgan, Talladega's coordinator of innovative learning, worked closely with superintendent Dr. Suzanne Lacey and the Digital Promise team to consider how the district could develop activities, curricula, and assessment that appealed to a broader range of students, with a focus on promoting inclusivity among students from low socioeconomic households and, more broadly, female students. Additionally, they focused on ensuring that such offerings were offered both consistently across its schools and classrooms and cumulatively (i.e., with coursework and activities building off each other). They leveraged existing leadership teams across schools to begin the process of designing Talladega's CT Pathway through a three-fold process:

Stage \#1: Why K-12 CT? In the first stage, Talladega clarified its vision for its own $\mathrm{K}-12$ computing pathway. Over a period of two months, the team met to identify the district's strengths, interests, and existing resources-as well as its gaps per grade level and across schools. They identified a few opportunities for computing in middle and high school, but little opportunity for students to build foundational computing skills outside of those classes. As a rural district, the Talladega community valued computing learning opportunities for students to thrive in the technologically evolving workforce and promote economic opportunity in the region. They resolved to integrate $\mathrm{CT}$ in each grade $\mathrm{K}-8$ and across disciplines.

Stage \#2: What do we mean by K-12 Computing? In the second stage, the Talladega district leadership team defined what new learning opportunities would be created across grade levels, courses, and schools. Here, Talladega tapped into Alabama's own Digital Literacy and Computer Science (DLCS) standards and relevant disciplinary standards to specifically define opportunities to integrate CT integration by grade level and within the context of specific subjects (i.e., math, ELA, and science). Talladega developed a "competency map" linking CT curricular specific activities and resources (e.g., Storytelling with Scratch in fourth grade ELA) to identify particular themes (e.g., digital storytelling) for integration, examining particular computing competencies (e.g., using algorithms, working with data, abstraction, etc.). 
Stage \#3: How to enact K-12 Computing for All? Talladega's third stage was most ambitious, addressing the nuts and bolts of school- and classroom-level change. Talladega shared their drafted pathway across the district, and leveraged Digital Promise's support to design surveys and interview protocols to gather teacher, administrative, student, and community feedback. Its pathway informed teacher Spring and then Fall professional development resources and supports, with sessions hereon tied to the specific competencies and activities identified on its pathway. Additionally, the district determined how they will measure the degree to which pathway implementation is making progress on their initial inclusivity goals, based upon student involvement in courses, student exit tickets on individual lessons, and classroom observation via "look fors" documents specific to the district's CT competencies. This last component of assessment is key in terms of how Talladega will update and add to its CT pathway for continuous improvement.

\section{Figure 5}

District Leader and Teacher Designing K-12 Opportunities for CT Integration in Talladega County

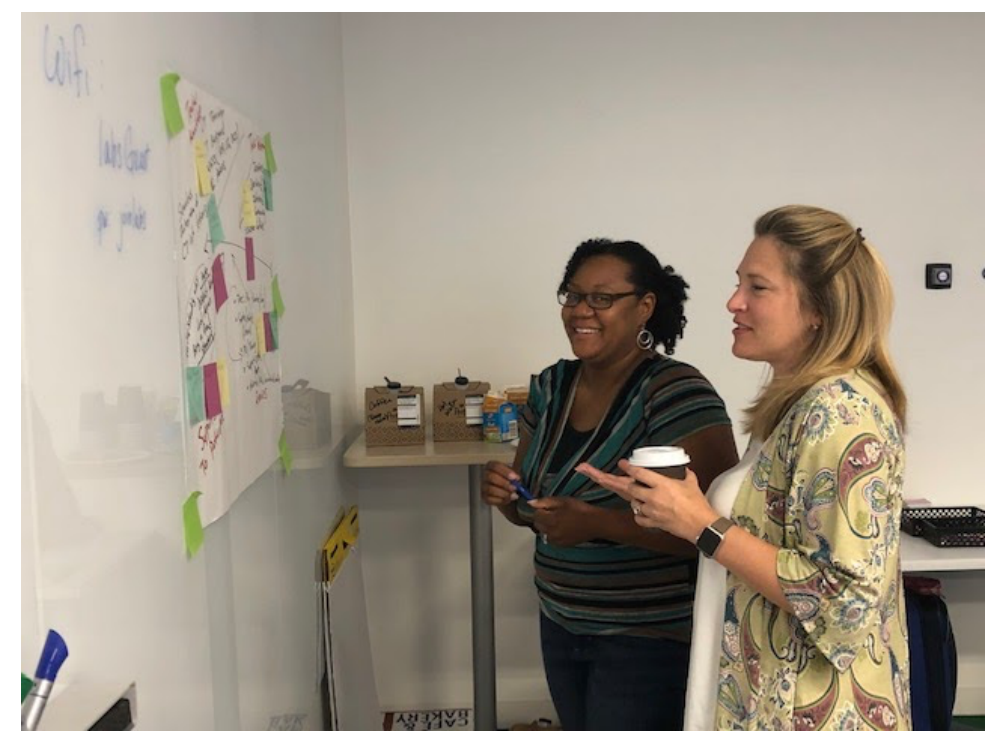

- Highlighted resource:

- Digital Promise's CT Pathways Toolkit (based on Talladega and other districts' piloting the process)

\section{Design for Inclusive Participation of Students Historically Marginalized from Computing}

lowa City Community School District decided to focus its computing pathway work on concerns about low participation in computing among Black students, Latinx students, and students designated as English learners. Inclusivity and equity challenges have arisen as the city has grown rapidly over the past decade, shifting from a rural and predominantly white college town to a burgeoning tech sector with a significant influx of Latinx families. 
In February 2020, lowa City participated in Digital Promise's Equity in the Driver's Seat convening, funded by the Carnegie Corporation of New York, in which researchers, practitioners, and other experts from the field discussed practitioner-identified, equitycentered problems of practice about computational thinking. During that time, the lowa City CT team took a hard look at their recent AP Computer Science course enrollment data, including demographic breakdowns. Upon examining the ecosystem in which the computing pathway is embedded, it became clear that there remain conditions (e.g., recruitment, access, pedagogy) leading to systemic inequities in the experiences of historically minoritized students at lowa City (Liberman \& Young, 2020).

In order to identify and address systemic inequities of computing offerings, the district formed an Inclusive CT Pathways Committee consisting of three high school, one middle school, and three elementary school teachers. The group started with the goal of identifying opportunities to create a more inclusive computing pathway for students through the development of tools like guidance documents, rubrics, and/or lesson plans. Before identifying opportunities in the pathway, the group began by conducting empathy interviews with their students as a way to consider different experiences, especially those of students historically excluded and marginalized students.

These empathy discussions would be valuable to talk to kids about what's

stopping them from taking computer science and what their fears are... [or] what is stopping them from taking these classes.

- Teacher, lowa City Community School District
At lowa City, teachers modified an empathy interview protocol developed by Digital Promise to conduct both empathy interviews and survey questions to learn about students' experiences with and decisions about participating in computing coursework. The empathy interview protocol moves teachers through a recognition of the need for empathy and user voice in design, noticing about themselves, conducting interviews, observing in

their classrooms, and reflecting on their experiences interviewing and what they learned during the interview (Ruiz et al., 2021). Teachers then spent two hour-long meetings debriefing what they learned from their students. In future meetings, they will use what they learned from the empathy interviews and surveys to think about and design supports, onramps, and more inclusive computing opportunities for their students.

- Highlighted resources:

- Equity in the Driver's Seat

- Empathy Interview Protocol

- Inclusive Integration of Computational Thinking 


\section{Engagement in Participatory and Iterative Design}

In the project Developing the Next Generation of Problem Solvers: Investigating the Integration of Computational Thinking into Preschool Mathematics and Science, funded by the National Science Foundation, we explore which CT skills resonate with young children's (ages 3-5) experiences, can be meaningfully linked math and science learning in preschool classrooms and children's homes, and can strengthen early learning more broadly. To develop integrated classroom and family learning resources, we brought together a diverse group of stakeholders to work in partnership to design and test playful learning activities and innovative digital games. Our co-design team included teachers and families from culturally diverse public preschool programs, curriculum designers, media developers at Curious Media, and learning scientists at Digital Promise, SRI Education and Edfinity. The team sought to take a participatory and inclusive approach to ensure all stakeholders shared their unique perspectives and insights to generate meaningful resources.

To begin the co-design process, our team of stakeholders came together to identify target content. Teachers and caregivers were invited to share experiences and highlight features of their classrooms and homes related to the project goals (e.g., the mathematics and science activities they typically plan and how children engage in them). Because CT is a new area in early childhood, CT researchers shared emerging CT definitions and initiated brainstorming sessions to generate possible examples relevant in preschool classrooms and homes. As an example, teachers and families suggested that everyday routines (e.g., bedtime or getting ready for school) could provide opportunities to embed CT because children could think and benefit from breaking (or decomposing) these tasks into smaller, more manageable subtasks.

\section{Figure 6}

Co-designing STEM-integrated CT Resources with PreK Teachers and Parents

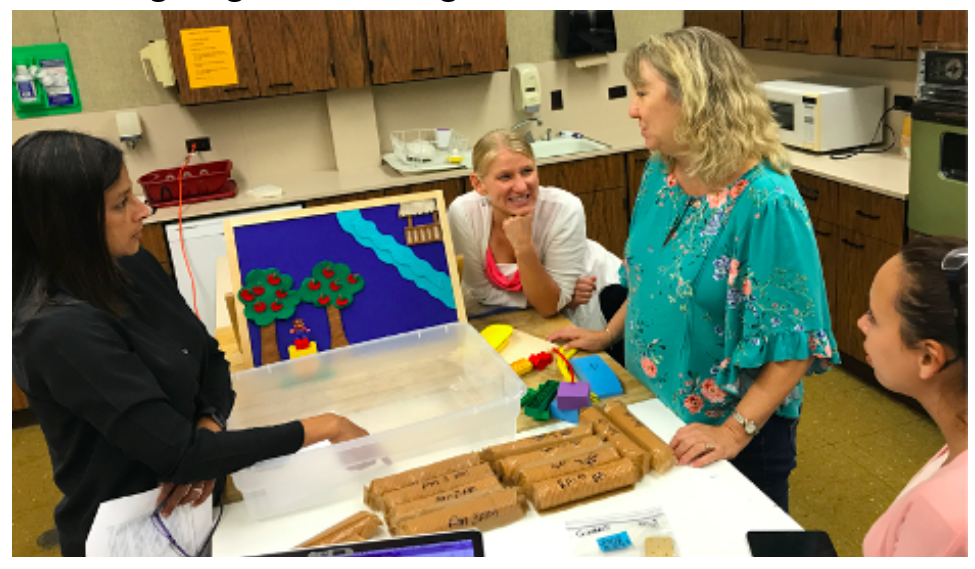


Figure 7

Our Co-design Team

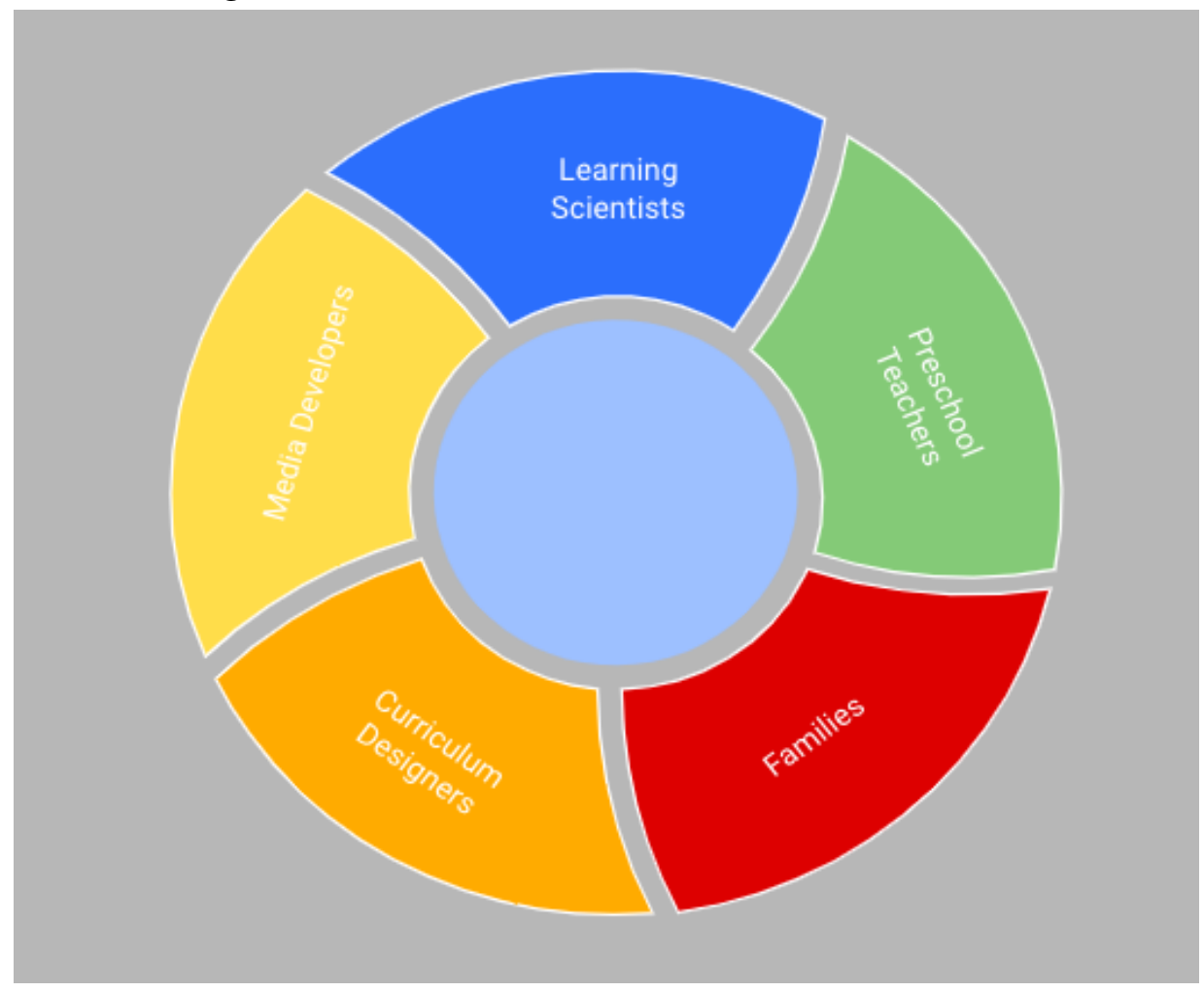

In addition, the co-design team developed and tested app prototypes to complement the hands-on activities by providing children with complementary opportunities to learn and practice CT skills. One app prototype focused on algorithmic thinking by inviting children to create a sequence of directions for a robot to deliver gifts to friends. This prototype closely aligned with one of the unplugged activities in which children followed a sequence of directions in order to navigate from one location to another on a large map. After multiple rounds of iteration informed by co-design discussions and findings from testing, the resulting "City Walk" game was developed and is currently included in the STEM-tastic Adventures app. This app retained the initial gift delivery premise that emerged during co-design and provides opportunities for children to identify a sequence of directions for the robot. The game progressively presents more complex sequences and scaffolds how to identify errors (testing and debugging) with the support of visual and audio feedback. We believe this inclusive codesign approach ultimately yielded resources that can be adopted and sustained more feasibly across home and school contexts. 
Figure 8

STEM-tastic Adventures App (available through the App Store and Google Play)

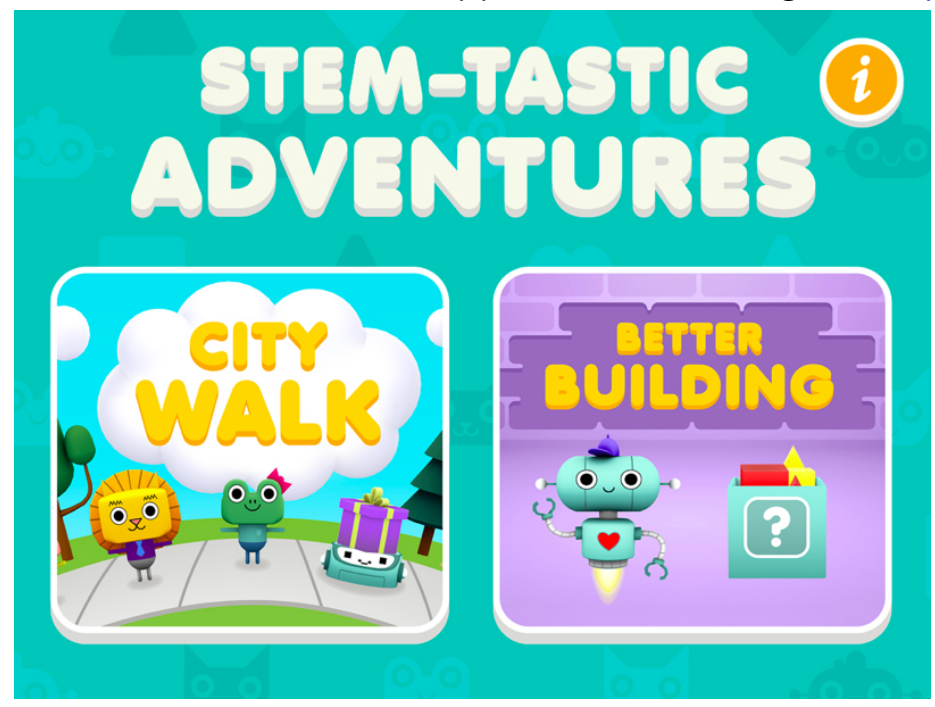

\section{Figure 9}

Young Learner Engaging in Computational Thinking through the STEM-tastic Adventures App (available through the App Store and Google Play)

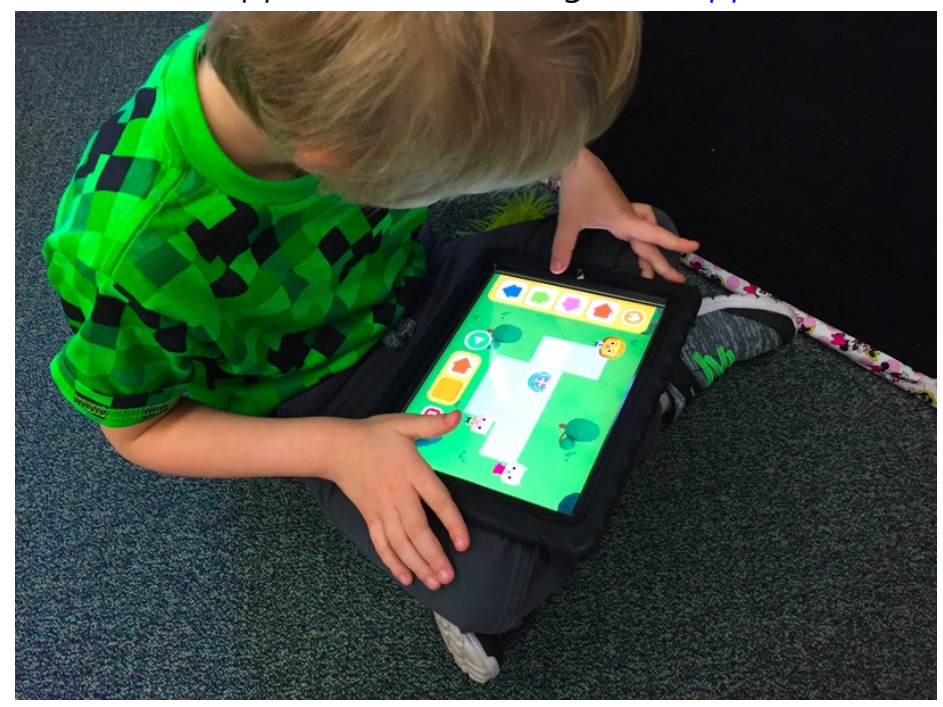

- Highlighted resource:

- STEM-tastic Adventures App 


\section{Digital Promise's Computational Thinking Resources}

\section{Reports}

\section{Computational Thinking for a Computational World}

This paper from December 2017 argues that computational thinking is both central to computer science and widely applicable throughout education and the workforce. It is a skillset for solving complex problems, a way to learn topics in any discipline, and a necessity for fully participating in a computational world

\section{Equity in the Driver's Seat}

This paper from July 2020 describes a collaborative process for developing practice-driven, equity-centered R\&D agendas. It accounts the equity-related challenges that on-the-ground staff were facing, while considering prominent gaps in existing research related to computational thinking.

\section{District Resources}

\section{Computational Thinking Pathways Toolkit}

This toolkit helps school and district leaders establish system-wide K-12 pathways that support equitable participation in computational thinking that is consistent across classrooms, cumulative from year to year, and competency-based.

\section{Teacher Resources}

\section{Computational Thinking for Next Generation Science Toolkit}

This toolkit was developed to help teachers reflect on their practice and identify opportunities to integrate computational thinking in middle school science. The content can be adapted to integrate computational thinking in other content areas, grade bands, and contexts.

\section{Computational Thinking Micro-credentials}

Digital Promise has created micro-credentials for Computational Thinking Practices. A micro-credential is a digital certificate that verifies an individual's competence in a specific skill or set of skills. To earn a micro-credential, teachers submit evidence of student work from classroom activities, as well as documentation of lesson planning and reflection. Microcredentials can be a useful tool for professional learning and/or credentialing pathways. 


\section{Empathy Interview Protocol}

When designing lessons and curricula that are inclusive of all students, it is important to begin by engaging with the students themselves to learn more about their experiences, motivations, and needs. Empathy interviews provide the opportunity to learn about the experiences of someone you are designing for in order to move forward with authentic student experiences at the center of lesson design.

\section{Student Resources}

\section{$\underline{\text { STEM-tastic Adventures App }}$}

A free app available through the App Store and Google Play to provide young children (ages 3-5) fun and engaging opportunities to learn CT and STEM.

\section{References}

Barr, V., \& Stephenson, C. (2011). Bringing computational thinking to K-12: what is Involved and what is the role of the computer science education community? ACM Inroads, 2(1), 48-54. https://doi.org/10.1145/1929887.1929905

Burke, Q., Roschelle, J., Angevine, C., O’Donnell, K.A., Smith, K., \& Weisgrau, J. (2019, March). Developing inclusive K-12 computing pathways for the League of Innovative Schools. Poster presentation at the 2019 RESPECT Conference, March 27th, Minneapolis, MN. https://doi.org/10.1109/RESPECT46404.2019.8985843

Dominguez, X., Grover, S. and Vahey, P. (2020). Enriching mathematics and science with computational thinking: Co-designing preschool activities with educators and parents. In Session, Integrating STEM \& Computing in PK-12: Operationalizing Computational Thinking for STEM Learning \& Assessment. In Proceedings of the 14th International Conference of the Learning Sciences, Nashville, TN. ISLS.

Lee, I., Grover, S., Martin, F., Pillai, S., \& Malyn-Smith, J. (2020). Computational thinking from a disciplinary perspective: Integrating computational thinking in $\mathrm{K}-12$ science, technology, engineering, and mathematics education. Journal of Science Education and Technology, 29(1), 1-8. https://doi.org/10.1007/s10956-019-09803-w

Liberman, B. \& Young, V. (2020). Equity in the driver's seat: A practice-driven, equity-centered approach for setting R\&D agendas in education. Digital Promise. https://doi.org/10.51388/20.500.12265/100

Margolis, J., Estrella, E., Goode, G., Holme, J. J., \& Nao, K. (2017). Stuck in the shallow end: Race, education, and computing. MIT Press.

Moll, L. C. (2015). Tapping into the "hidden" home and community resources of students. Kappa Delta Pi Record, 51(3), 114-117. https://doi.org/10.1080/00228958.2015.1056661 
National Academies of Sciences, Engineering, and Medicine (NASEM) 2021. Cultivating Interest and Competencies in Computing: Authentic Experiences and Design Factors. The National Academies Press. https://doi.org/10.17226/25912

Nesiba, N., Pontelli, E., \& Staley, T. (2015). DISSECT: Exploring the relationship between computational thinking and English literature in K-12 curricula. 2015 IEEE Frontiers in Education Conference (FIE), 1-8. https://doi.org/10.1109/FIE.2015.7344063

Pinkard, N., Erete, S., Martin, C. K., \& McKinney de Royston, M. (2017). Digital Youth Divas: Exploring narrative-driven curriculum to spark middle school girls' interest in computational activities. Journal of the Learning Sciences, 26(3), 477-516. https://doi.org/10.1080/10508406.2017.1307199

Roschelle, J., Burke, Q., Ruiz, P., \& Santo, R. (2021). Towards CS/CT pathways: Creating guidance documents as district leadership development. Policy Futures in Education, Special Issue of Equity in 21st Century Computer Science Education (Eds., J. Childs, A. Leftwich, and J. Flapan).

Ruiz, P., Mills, K., Coenraad, M., \& Burke, Q. (2021). Teacher and student empathy interviews as an instrument for considering more inclusive $K-12$ computing pathways. The Annual Conference on Research in Equity and Sustained Participation in Engineering, Computing, and Technology Conference, May 23-27, 2021. Virtual.

Ryoo, J. J. (2019). Pedagogy that supports computer science for all. ACM Transactions on Computing Education (TOCE), 19(4), 36. https://doi.org/10.1145/3322210

Settle, A., Franke, B., Hansen, R., Spaltro, F., Jurisson, C., Rennert-May, C., \& Wildeman, B. (2012). Infusing computational thinking into the middle- and high-school curriculum. Proceedings of the 17th ACM Annual Conference on Innovation and Technology in Computer Science Education - ITiCSE '12. https://doi.org/10.1145/2325296.2325306

Waterman, K. P., Goldsmith, L., \& Pasquale, M. (2020). Integrating computational thinking into elementary science curriculum: An examination of activities that support students' computational thinking in the service of disciplinary learning. Journal of Science Education and Technology, 29(1), 53-64. https://doi.org/10.1007/s10956-019-09801-y 